\title{
Neurometabolic coupling in the vegetative and minimally conscious states: preliminary findings
}

\author{
M R Coleman, D K Menon, T D Fryer, J D Pickard
}

J Neurol Neurosurg Psychiatry 2005;76:432-434. doi: 10.1136/jnnp.2004.045930

The objective of this study was to determine the integrity of the homoeostatic coupling relationship between neuronal electrical function and cerebral metabolism in the vegetative and minimally conscious states. Ten patients who met recognised diagnostic criteria (six in the vegetative state and four minimally conscious) were investigated using simultaneous electroencephalography and positron emission tomography. It was found that the coupling between neuronal electrical activity and regional glucose metabolism was preserved in all the minimally conscious patients but was absent in all the vegetative state patients. Our preliminary results suggest patients in the vegetative state may endure an impaired coupling relation between neuronal electrical function and cerebral energy metabolism.

A lthough behaviourally the presence of awareness distinguishes the vegetative and minimally conscious states, the pathophysiological basis of this distinction between the two groups remains unclear. Metabolic imaging has failed to find an increase in overall cerebral metabolic rates as a comatose patient regains consciousness. ${ }^{1}$ Similarly, structural brain imaging and pathological investigations have failed to find changes that are specific to either condition or discriminate between them. Many patients have diffuse axonal injuries, often giving rise to degenerative white matter changes throughout the cerebral hemispheres and brainstem. ${ }^{2}$ Alternatively, many patients remaining in these states of impaired consciousness have experienced cerebral hypoxia or ischaemia leading to diffuse or multifocal laminar necrosis, thalamic and other focal lesions. ${ }^{2}$ A study by Jennett and colleagues $^{3}$ compared the neuropathological differences between severely disabled patients (including the minimally conscious state) and those in the vegetative state. They found similar structural lesions in both groups, but these were less severe in the severely disabled and minimally conscious patient groups. Electrophysiological investigations are less complete with insufficient data currently available about the electroencephalogram (EEG) presentation in minimally conscious patients. ${ }^{4}$ In the vegetative state the EEG is described as "typically slow wave activity", ${ }^{4}$ although this is likely to vary depending upon the cause of the injury.

Recent attempts to determine the pathophysiological distinction between the vegetative and minimally conscious states have focused upon the integrity of cortico-cortical and thalamocortical networks. However, no attempts have been made to determine the integrity of the homoeostatic coupling relation between neuronal electrical function and cerebral metabolism.

\section{METHOD}

Six patients (three men, three women; mean age 51 years, range 20-67) who met the diagnostic criteria for the vegetative state ${ }^{4}$ and four patients (two men, two women; mean age 54 years, range 40-70) who met the diagnostic criteria for the minimally conscious state $^{5}$ were studied simultaneously with a 19-channel EEG and positron emission tomography (PET) using ${ }^{18} \mathrm{~F}$-2-fluoro-2-deoxy-D-glucose.

The patients were recruited from local neurological wards over a 12 month period. The study was approved by the Cambridge Regional Ethics Committee (LREC 99/391) and all patients were recruited following informed assent from the next of kin. The patients were assessed by a consultant neurologist and categorised according to the Royal College of Physicians' guidelines ${ }^{4}$ and the Aspen workgroup. ${ }^{5}$ The neurologist was blinded to the purpose of the study. Five patients meeting the criteria defining the vegetative state and two patients meeting the criteria defining the minimally conscious state had non-traumatic injuries. These included subarachnoid haemorrhage $(\mathrm{n}=3)$ and intracerebral haemorrhage $(n=4)$. Three patients (minimally conscious $\mathrm{n}=2$ ) had traumatic brain injuries. These included diffuse axonal injury $(\mathrm{n}=2)$ and subarachnoid haemorrhage $(\mathrm{n}=1)$.

PET studies were undertaken on a General Electric Advance scanner (GE Medical Systems, Milwaukee, USA). Emission data were acquired in three dimensional mode following a 30 second intravenous bolus of $74 \mathrm{MBq}$ of ${ }^{18} \mathrm{~F}-2$ fluoro-2-deoxy-D-glucose. Just prior to emission data acquisition, a 10 minute transmission scan was done using rotating germanium-68 rods $(2 \times \sim 300 \mathrm{MBq})$. These data, in combination with those from a 60 minute blank scan acquired on the same day, were used to correct the emission data for photon attenuation. The images were reconstructed into $2.34 \times 2.34 \times 4.35 \mathrm{~mm}$ voxels using the PROMIS $3 \mathrm{D}$ filtered back projection algorithm, with corrections applied for attenuation, scatter, randoms, and dead time. During reconstruction, a Hanning filter cut-off at the Nyquist frequency was used transaxially, but no apodisation was applied axially. Emission images were smoothed using an isotropic $4 \mathrm{~mm}$ Gaussian filter. The cerebral metabolic rate of glucose was calculated using the Patlak plot with a lumped constant of 0.52 and PET data were processed using Analyze AVW 5 (Biomedical Imaging Resource, Mayo Foundation, Rochester, MN, USA). Parametric maps were analysed using 19 anatomical regions of interest, which corresponded to the position of the EEG electrodes. All regions of interest were cortical.

EEG recordings were made (bandpass $0.1-70 \mathrm{~Hz}$ ) from 19 scalp electrodes placed according to the International 1020 system. From each recording, multiple artefact free segments of the EEG were selected and a fast Fourier transform was performed to determine the bandpower distribution across the frequency range $1-40 \mathrm{~Hz}$. The square root of average power was then calculated for each frequency

Abbreviations: $\mathrm{EEG}$, electroencephalogram; PET, positron emission tomography; PRI, power ratio index 
band: delta $(2-3.8 \mathrm{~Hz})$, theta $(4.0-7.8 \mathrm{~Hz})$, alpha (8.0$12.8 \mathrm{~Hz})$, and beta $(13.0-29.8 \mathrm{~Hz})$. The ratio of slow to fast wave EEG activity, referred to as the power ratio index (PRI) was calculated as the ratio of percentage power in the delta and theta frequency bands (slow wave activity) to the percentage power in the alpha and beta frequency bands (fast wave activity). Percentage power was defined as the relative value of the square root of average power within each frequency band to that of the whole frequency spectrum.

All patients were investigated within six months of injury. At present no information on outcome is available.

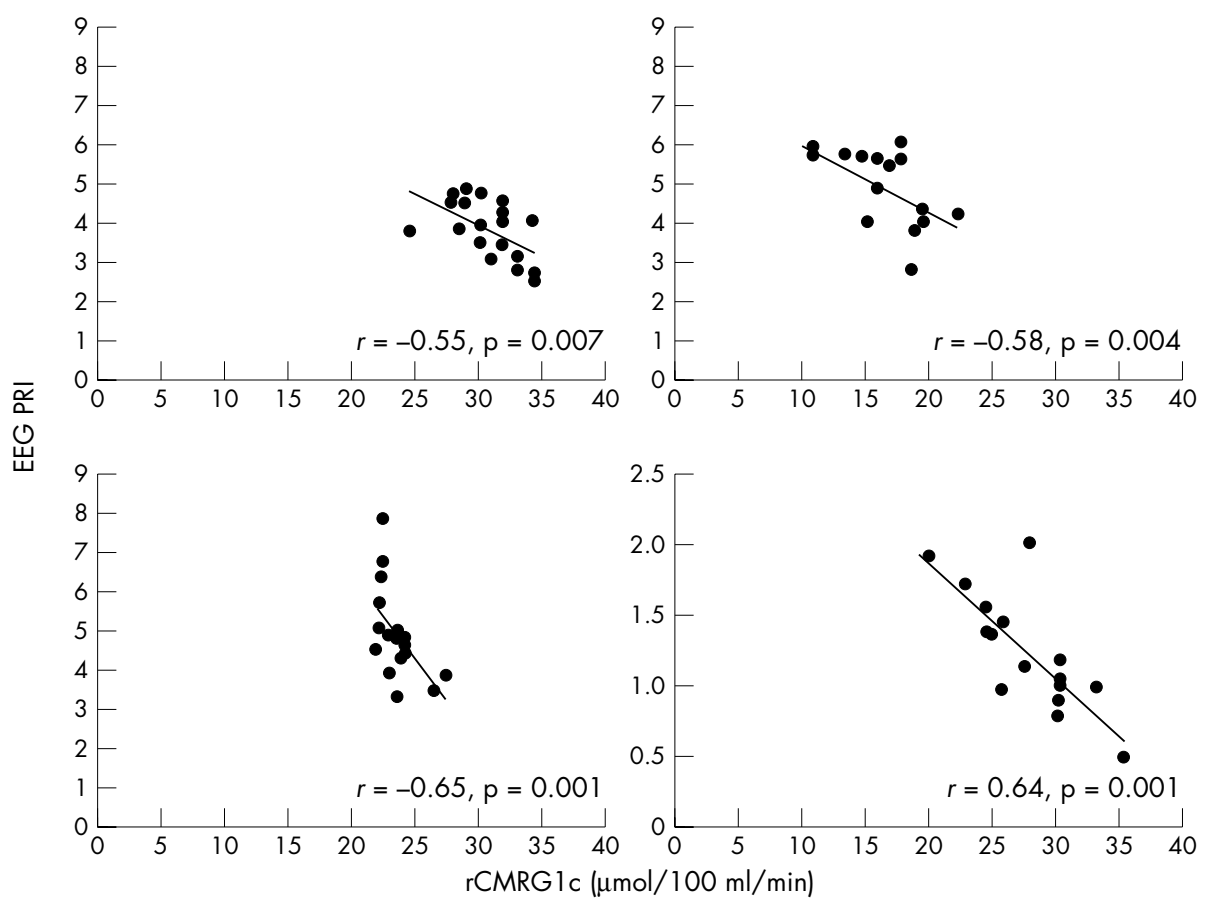

Figure 1 Correlation between the electroencephalogram (EEG) power ratio index (PRI) and rCMRG c (regional cerebral metabolic rate for glucose) in the minimally conscious patient group. The graphs show weak but significant $(p<0.01)$ correlations between neuronal electrical activity and regional glucose metabolism in all four minimally conscious patients.
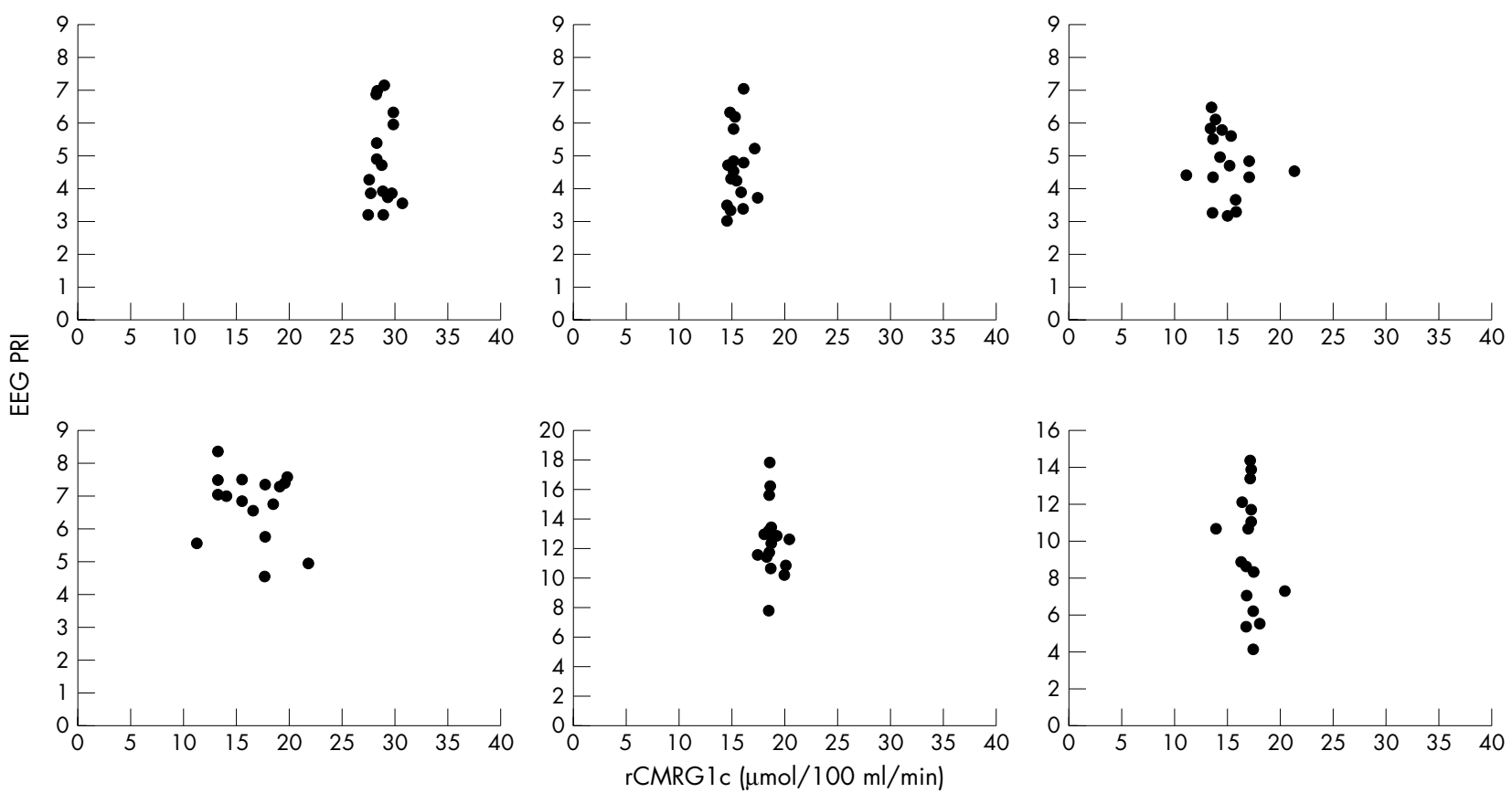

Figure 2 Correlation between electroencephalogram (EEG) power ratio index (PRI) and rCMRG1c (regional cerebral metabolic rate for glucose) in the vegetative state patient group. None of the six vegetative patients investigated showed a significant relationship between neuronal electrical activity and regional glucose metabolism. 


\section{RESULTS AND DISCUSSION}

We found that the regional cerebral metabolic rate of glucose metabolism did not vary significantly between patients in the vegetative and minimally conscious groups $(p=0.4)$. The EEG, however, showed an encephalopathic background in the vegetative state and a mixed background of theta and alpha frequencies in the minimally conscious state group. Similarly, the vegetative group showed a significantly higher PRI in comparison with the minimally conscious group $(p<0.05)$, indicating a large degree of slow wave activity in the vegetative group. The correlation between EEG PRI and regional glucose metabolism was preserved in all the minimally conscious patients (fig 1), but was absent in all the patients in the vegetative state (fig 2).

Our results suggest the important homoeostatic relation between neuronal electrical function and cerebral metabolism was impaired across regions of the cerebrum in our patients in the vegetative state group but was preserved in the minimally conscious state group. Future corroboration may raise important opportunities for therapeutic treatment of impaired neurometabolic coupling.

\section{ACKNOWLEDGEMENT}

This work was supervised by Dr Simon Boniface, Department of Clinical Neurophysiology, Addenbrooke's Hospital, Cambridge, who sadly passed away on 27 November 2003.
Authors' affiliations

M Coleman, Department of Clinical Neurophysiology, Addenbrooke's Hospital, Cambridge, UK

M Coleman, D Menon, T Fryer, J Pickard, Wolfson Brain Imaging

Centre, University of Cambridge, UK

This work was funded by the Smiths Charity.

Competing interests: none declared

Correspondence to: M R Coleman, Cambridge Coma Study Group, Box 124, Addenbrooke's Hospital, Cambridge, CB2 2QQ, UK;

mrc30@wbic.cam.ac.uk

Received 18 May 2004

In revised form 1 July 2004

Accepted 6 July 2004

\section{REFERENCES}

1 Laureys S, Lemaire $\mathrm{C}$, Maquet $\mathrm{P}$, et al. Cerebral metabolism during vegetative state and after recovery to consciousness. J Neurol Neurosurg Psychiatry 1999;67:121.

2 Adams JH, Graham DI, Jennett, B. The neuropathology of the vegetative state after an acute brain insult. Brain 2000;123:1327-38

3 Jennett B, Adams JH, Murray LS, et al. Neuropathology in vegetative and severely disabled patients after head injury. Neurology 2001;56:486-90.

4 Royal College of Physicians (UK). The vegetative state: guidance on diagnosis and management. A report of a working party of the Royal College of Physicians. Clin Med 2003:2:249-54.

5 Giacino JT, Ashwal S, Childs N, et al. The minimally conscious state: definition and diagnostic criteria. Neurology 2002;58:349-53. 